\title{
Lucha política en Twitter del ciberespacio. Partidos políticos en Perú (2019-2020)
}

Recibido: 27 de septiembre de 2020 • Aprobado: 3 de diciembre de 2020

https://doi.org/10.22395/ojum.v20n42a20

\author{
Cluber Fernado Aliaga Lodtmann \\ Universidad Jaime Bausate y Meza, Lima, Perú \\ caliaga@bausate.edu.pe \\ https://orcid.org/0000-0001-9819-3362
}

\section{RESUMEN}

El objetivo del presente trabajo fue conocer la forma en que se desarrolló la lucha política en la red social Twitter del ciberespacio desde junio de 2019 hasta junio de 2020. En este contexto, se determinó el nivel de adhesión de la población, la eficacidad en el logro de nuevos seguidores, el nivel de producción informativa, la efectividad, el tipo de los mensajes difundidos, los objetivos aparentes, las temáticas más recurrentes, las alianzas deducidas y el mapa de conflictividad de los partidos políticos. Se parte de una breve revisión de la teoría y de investigaciones previas relacionadas. Los resultados muestran un desarrollo asimétrico, irregular y nada homogéneo de la lucha de los partidos políticos peruanos. Se identifican las principales características de la lucha política en el ciberespacio en el Perú mediante el empleo de la red social Twitter por diez de los veinticuatro partidos políticos inscritos ante el JNE en junio de 2019. De diez partidos seleccionados cuatro no han registrado actividad comunicativa (PAP, AP, SP y PPC); dos, poca actividad (FP y PM); dos, mediana actividad (PNP y FA); y dos, alta actividad (APP y C). El PNP y FP son los que más confrontan, en tanto que la mayoría trata de evitar el antagonismo evidente, y PM evita críticas o comentarios contra partidos o líderes políticos.

Palabras clave: Twitter; comunicación política; ciberpolítica; redes sociales; internet. 


\section{Political Struggle on Twitter's Cyberspace. Political Parties in Peru (2019-2020)}

\section{ABSTRACT}

The objective of this work was to know the way in which the political struggle developed in the cyberspace social network Twitter in the period June 2019 - June 2020, determining the level of adherence of the population, effectiveness in the achievement of new followers, level of informative production, effectiveness and type of the disseminated messages, apparent objectives, most recurrent themes, deduced alliances, and the map of conflict of the political parties. It begins with a brief review of the theory and related previous research. The results show an asymmetric, irregular and not homogeneous development of the struggle of the Peruvian political parties. The main characteristics of the political struggle in in the Peruvian cyberspace are identified through the use of the Twitter social network by 10 of the 24 political parties registered with the JNE as of June 2019. Of the 10 selected parties, four have not registered communicative activity (PAP, AP, SP and PPC); two, little activity (FP and PM); two, medium activity (PNP and FA) and two, high activity (APP and C). The PNP and FP are the most confrontational, while the majority try to avoid obvious antagonism, and one (PM) avoids criticism or comments against parties or political leaders.

Keywords: Twitter; political communication; cyber politics; social networks; internet.

\section{Combate político no Twitter do espaço cibernético. Partidos políticos no Peru (2019-2020)}

\section{RESUMO}

O presente trabalho tem como objetivo conhecer a forma como se desenvolveu a luta política na rede social Twitter do espaço cibernético entre os meses de junho de 2019 a junho de 2020. Nesse contexto, foi determinado o nível de adesão da população, a eficácia na obtenção de novos seguidores, o nível da produção informativa, a efetividade, o tipo das mensagens difundidas, os objetivos aparentes, os assuntos mais frequentes, as alianças deduzidas e o mapa do conflito entre os partidos políticos. Parte-se de uma breve revisão da teoria e de pesquisas prévias relacionadas. Os resultados mostram um desenvolvimento assimétrico, irregular e nada homogêneo da luta dos partidos políticos peruanos. Se identificam as principais características do embate político no espaço cibernético no Peru por meio da rede social Twitter nos dez dos vinte e quatro partidos políticos inscritos na JNE em junho de 2019. Dos dez partidos escolhidos, quatro não tinha registrado atividade comunicativa (PAP, AP, SP e PPC); dois, pouca atividade (FF e PM); dois, meia atividade (PNP e FA); e dois, alta atividade (APP e C). O PNP e FP são os que mais confrontam, enquanto que a maioria busca evitar o antagonismo evidente, e PM evita críticas ou comentários contra partidos ou líderes políticos.

Palavras clave: Twitter; comunicação política; ciberpolítica; redes sociais; internet. 


\section{INTRODUCCIÓN}

El presente artículo deriva del interés personal e investigativo del autor y su compromiso por desarrollar proyectos de investigación en la Universidad Jaime Bausate y Meza. El objetivo es determinar las principales características de la lucha política librada en el ciberespacio a partir de una muestra de los partidos políticos peruanos mediante el empleo de la red social Twitter en el período comprendido entre junio 2019 y junio 2020.

El período de estudio se caracterizó por haber registrado una intensa actividad política. En esta actividad se destacan: i) el cierre del Congreso de la República dispuesto por el poder ejecutivo, producida el 30 de septiembre de 2019, por la causal de denegatoria de la tercera cuestión de confianza presentada, que se dio mediante el Decreto Supremo 165-2019-PCM (2019); ii) la convocatoria y posterior realización de las elecciones congresales extraordinarias 2020, desarrolladas el domingo 26 de enero de 2020, que planteó el surgimiento de nuevos actores políticos; y iii) el inicio del aislamiento social obligatorio en el Perú para hacer frente a la pandemia de la COVID-19 el lunes 16 de marzo de 2020. Esta medida se establece mediante Decreto Supremo 044-2020-PCM (2020), que declara el estado de emergencia nacional por las circunstancias que afectan la vida de la nación a consecuencia del brote de la COVID-19. Con este decreto se restringe el ejercicio de los derechos constitucionales relativos a la libertad y la seguridad personales, la inviolabilidad del domicilio y la libertad de reunión y de tránsito en el territorio nacional.

Se ha partido de la revisión en profundidad de la literatura previa sobre el tema y se destacaron los hallazgos más relevantes. Asimismo, se registraron todos los mensajes emitidos por los partidos políticos seleccionados durante un mes dentro del periodo del estudio (junio de 2019-junio de 2020). Se tomó nota del número de seguidores iniciales y finales, la producción informativa, los mensajes más efectivos, los tipos de mensajes que tuvieron mayor acogida, la determinación de los objetivos aparentes de los partidos políticos, las temáticas más recurridas, la deducción de las alianzas políticas, así como la determinación del mapa de conflictividad de los partidos políticos en el Perú.

Se optó por el estudio de la red social Twitter debido a que esta permite a los ciudadanos intervenir activamente en la lucha política. De esta manera, los ciudadanos generan sus propios mensajes, emiten sus opiniones o manifiestan sus acuerdos o desacuerdos a los mensajes difundidos por los partidos políticos o líderes de las mismas mediante las opciones de "me gusta", comentar y compartir.

Esta incorporación de los ciudadanos en la emisión, interpretación y difusión de los mensajes políticos a través de las redes sociales, con la participación en la intermediación y difusión informativa conjuntamente con el periodismo político, 
ha motivado a Chadwick (2017) a proponer la sustitución del tradicional concepto de ciclo de noticias por el de ciclo de información política.

Al mes de junio 2019, las organizaciones políticas (OP) que figuraban en el registro oficial del Jurado Nacional de Elecciones (JNE) como partidos políticos sumaban veinticuatro. Sin embargo, en las elecciones presidenciales del domingo 10 de abril de 2016 (primera vuelta) y el domingo 5 de junio de 2016 (segunda vuelta) solo participaron diez, de los cuales ocho partidos pasaron la valla electoral'1. Por otro lado, el Partido Político Orden (0,4 \%) de Antero Flores-Araoz y Perú Posible (1,3\%) de Alejandro Toledo perdieron su inscripción por no pasar la valla electoral (El Comercio, 2019).

El estudio permitió la comprensión de la forma en que se ha venido dando la lucha política en el Perú, a nivel del ciberespacio, mediante el empleo de la red social Twitter, a lo que algunos denominan "ciberpolítica". Se identificaron aspectos favorables y desfavorables, así como las principales características detectadas, lo que nos permitió formular algunas propuestas para la mejora de la comunicación política a nivel de los partidos políticos e identificar temas de interés para futuras investigaciones sobre este campo.

La investigación se orientó, en específico, al registro de los mensajes divulgados por los partidos políticos seleccionados en sus páginas oficiales de Twitter como muestra. Estos fueron procesados mediante el empleo de una base de datos en Excel. La investigación es de tipo aplicada y de enfoque mixto (cuali-cuantitativa), su nivel es exploratorio y descriptivo y su diseño es ex post facto, no experimental y de corte.

La población de estudio comprendió a los partidos políticos reconocidos oficialmente por el JNE-Perú, al mes de junio de 2019, que sumaban veinticuatro organizaciones. De estas se seleccionó una muestra por conveniencia de diez partidos políticos, considerados como los más importantes del Perú a criterio del investigador. Se tomó en cuenta los votos que lograron captar y el número de militantes afiliados en su padrón oficial y de seguidores que tienen en la red social Twitter. Se incluyó al Partido Morado, inscrito el mes de abril del 2019, por su buen desempeño en las elecciones generales 2016 con la figura del candidato Julio Guzmán. Los partidos políticos seleccionados para nuestro estudio fueron: Fuerza Popular (FP), Frente Amplio (FA), Partido Aprista Peruano (PAP), Acción Popular (AP), Alianza para el Progreso (APP), Contigo (C), Partido Popular Cristiano (PPC), Partido Nacionalista Peruano (PNP), Somos Perú (SP) y Partido Morado (PM).

Valla electoral se refiere al requisito que deben cumplir los partidos políticos que participan en un proceso de elecciones generales en el Perú para mantener su inscripción como partido y seguir en el proceso electoral; es lograr un mínimo de votos en el cómputo general del $5 \%$. 


\section{LUCHA POLÍTICA Y CIBERESPACIO}

\subsection{Lucha Politica}

Plejánov (2017) entiende que la lucha política expresa toda lucha de clases, lo cual nos da la idea de que la lucha política comprende el enfrentamiento entre, por lo menos, dos grupos antagónicos dentro de una sociedad que pugnan por sus propios intereses.

Bovero (1997) señala que no todo conflicto o lucha es política. Para que ello ocurra, se requiere que el conflicto verse sobre las estructuras o reglas del orden político. En este sentido, afirma:

La conservación o transformación de las formas de convivencia social, el mantenimiento o la conquista del poder ya que este es el medio necesario para ello. Así la lucha política, sería el conflicto por el poder de imponer un cierto orden político. (Bovero, 1997, p. 101)

Velásquez (2013) sugiere que la sociedad es un espacio en la que convergen múltiples relaciones de poder, esto es, es un escenario permanente de la lucha política. En este sentido, rescata la frase de Clausewitz: "la guerra es la política librada por otros medios y que la misma política es la continuación de la guerra por otros medios" (Velásquez, 2013, p. 177). Por nuestra parte reconocemos que el conflicto siempre ha estado presente en las sociedades humanas.

Para nosotros la lucha política es un conflicto evidente o no entre por lo menos dos grupos antagónicos en una sociedad que pugnan por la obtención del poder, su ejercicio o su preservación. Para ello, emplean como medios la persuasión o la sumisión de la población.

A nivel del ciberespacio, se reproduce esta pugna por el poder entre los grupos antagónicos, lo que evidencia diversidad en las relaciones sociales. Esto se opone a la política paradigmática que se orienta al orden y la coexistencia pacífica (Canetti, 1960; Mouffe, 1999). Por otra parte, Condori (2017) nos sugiere que "la política es ambivalente: por un lado, lucha y, por otra integración" (p. 23). Como lucha, evidencia el antagonismo entre dos o más grupos; como integración, se esfuerza por lograr la cohesión al interior de cada grupo político.

Internet, debido a su capacidad para ampliar la comunicación humana, es utilizada como un arma poderosa para la lucha política, consolidar liderazgos, propuestas, así como para aplicar tácticas y estrategias políticas (Cotarelo, 2013). La lucha política hoy se desarrolla en el ciberespacio, a través de las redes sociales. Dentro de estas se destaca Twitter por sus facilidades para el debate político y los esfuerzos para ganar la adhesión, el apoyo o respaldo de la población a las candidaturas, propuestas o decisiones políticas. Este esfuerzo es realizado por comunicadores políticos que forman parte de los gabinetes de comunicación política al interior de cada partido político. 
Este gabinete está integrado por periodistas, expertos en mercadeo político, psicólogos sociales, sociólogos, politólogos y técnicos en informática que vienen sentando las bases de una nueva especialidad profesional, la del comunicador político, cuyo trabajo no el mismo que el del periodismo político.

La lucha política emplea la comunicación política que, según Aira (2015), es "toda comunicación que tiene por objeto la política" (p. 32). El comunicador político procura optimizar los resultados de toda comunicación política en función de los objetivos del partido. En este sentido, debe "explorar todas las oportunidades y de anticiparse a todos los obstáculos" (Aira, 2015, p. 47). Esto hace que la comunicación política sea clave para "casi todo" en la lucha política, como lo hiciera John Fitzgerald Kennedy, uno de los primeros políticos que empleó el marketing bajo la premisa de que "el futuro no es un regalo, sino una conquista" (Aira, 2015, p. 48).

Para Aguilar (2016) los partidos políticos "son organizaciones emanadas de la sociedad que buscan representar a la misma, incluyendo su participación para perdurar en el poder" (p. 10). Por afiliación libre, logran otorgar poder a sus líderes o representantes, así como captar a los votantes, quienes se consideran en el marketing político y la publicidad como consumidores "que tiene que elegir entre una gama de productos; en este caso, líderes y partidos políticos" (Aira, 2015, p. 32).

El periodismo político ha sido tradicionalmente la "práctica informativa que se ocupa de la actividad de los gobiernos, los partidos y las organizaciones políticas, las campañas políticas, las elecciones y todos los acontecimientos que están relacionados con la esfera pública" (Condori, 2017, p. 35). Además, comprende los aspectos organizacionales, las protestas y las manifestaciones sociales. Mientras tanto, el comunicador político busca influir y persuadir fundamentalmente a la población para apoyar a determinarlo candidato, propuesta política o decisión que se quiera implementar. En este sentido, apela a estrategias de publicidad y marketing político, hace uso de los conocimientos de la psicología y sociología y prepara mensajes adecuados para cada uno de sus distintos públicos como jóvenes, hombres, mujeres, adultos, profesionales, religiosos, etc., a fin de ganar la mayor adhesión posible. Entre sus actores se encuentran los políticos, los líderes de opinión, las élites, los estrategas de la comunicación política, los medios de comunicación y los periodistas.

Sumado a lo anterior, Velásquez (2013) señala que el espacio social es delineado por las acciones y decisiones que el poder y su reparto va adoptando, donde la distinción amigo/enemigo es "fundamental para definir lo político" (p.193). Lo político sigue vinculado a la lucha entre grupos de aliados y simpatizantes por cuotas de poder.

\subsection{Ciberespacio}

Según Aparici (2010), "[e]l ciberespacio es un "no lugar" [...] lorganizado principalmente] por los países del primer mundo que tienen el control y el poder informacional de la 
web" (pp. 30-31). Nosotros acotamos que, más que en los países, el poder informacional se encuentra en manos de empresas privadas y pocos propietarios que detentan el poder informativo global en el ciberespacio. Según Aparici (2010), este es la "materia prima a la información de cualquier naturaleza" (p. 22).

La creación y el desarrollo del ciberespacio es una condición básica del nuevo escenario mundial, en el que el comercio, la educación, la interculturalidad y las relaciones humanas se están transformando para dar lugar a un "nuevo orden económico, social y cultural globalizado" (Aparici, 2010, p. 127). El ciberespacio constituye un nuevo escenario en el que se desarrollan las relaciones sociales. Para ingresar a ella es necesario un medio tecnológico que permita las comunicaciones en línea, como computadoras, teléfonos inteligentes y otros que permitan el flujo de información y la interacción humana.

Internet es la pieza clave de la denominada "sociedad de la información", "ciberespacio" y "comunidad global virtual". Su empleo a nivel global ha modificado "las relaciones económicas, políticas, sociales y, muy especialmente, las personales" (Barrio, 2017, p. 9). Un aspecto que revela el impacto del internet en la vida humana es el surgimiento de los cibercrímenes, la ciberguerra, el ciberterrorismo, etc., que amenazan a todos los que interactúan en el ciberespacio. Estos pueden "desestabilizar un Estado" y llegar a "paralizar importantes instituciones de un país" (Barrio, 2017, p. 15).

Para nosotros, el ciberespacio es el ámbito en el que se desarrolla la comunicación humana y un nuevo tipo de interacción social muy dinámica que surge por las ventajas y posibilidades que permiten las nuevas tecnologías de la información, la comunicación y el internet. Nos referimos a la comunicación digital, los negocios en línea, las redes sociales, así como todos los aplicativos y usos que tiene y puede tener internet.

Podemos afirmar que la sociedad humana en general está pasando a desarrollar sus relaciones e interacciones a través de las computadoras y tecnologías de la información y comunicación, por lo que se demanda a estos los máximos niveles de seguridad (Cortés, 2004). Esta demanda de mayor seguridad en este nuevo escenario de la interacción social viene generando el desarrollo de tecnologías de ciberseguridad, sistemas de prevención y sanción penal para los cibercrímenes y el desarrollo de una ciberpolicía que pueda actuar en la prevención y represión de los cibercrímenes a nivel global.

Las redes sociales también sirven para generar lazos interpersonales, brindar apoyo, información y un sentimiento de pertenencia e incluso identidad social (Wellman, 2001).

Según López-Rabadán, López-Mery y Doménech-Fabregat (2016), Twitter se presenta como una plataforma autónoma y favorable para la difusión de mensajes políticos 
de manera directa, instantánea, interactiva, y ya filtrada hacia seguidores previamente interesados. Estas características sirven a los propósitos de los líderes y partidos políticos, porque permiten el diálogo con los usuarios de manera bidireccional y horizontal y con los inconvenientes que ello pueda representar.

Marreros (2018) señala que la participación de los usuarios de las redes sociales los ha convertido en una fuente de información (prosumer), lo que constituye un contrapeso a los medios tradicionales, ya que estos no solo reciben, sino que también producen y comparten información. Por su parte, Mejía (2015) señala que las campañas electorales ahora se desarrollan en las redes sociales. En el Perú se registró un desarrollo importante. En el año 2010 comenzó con un incipiente uso por parte de las candidatas a la Alcaldía de Lima, Susana Villarán y Lourdes Flores. Pero este se consolidó en el 2013 en la campaña de revocatoria de la alcaldesa de Lima, en la que demostró ser un medio efectivo para conservar el poder.

Los debates públicos en el ciberespacio, a nivel de los partidos políticos peruanos, registran violencia en sus expresiones a favor o en contra de las distintas posiciones políticas (Meléndez, 2012), en especial por parte de los troll. Los troll son operadores políticos encubiertos que opinan a favor y en contra de los opositores. Para ello, emplean insultos y ofensas personales para descalificar a los contrarios; entre ellos, se suele identificar a los fujitroll y los caviares. Los primeros son todos los que en una red social se dedican a atacar a los opositores políticos del fujimorismo, mientras que los segundos son personas que, pese a tener un nivel económico superior al de las mayorías, tienen un discurso de izquierda.

Marcos García (2018) reconoce que el público participa en la creación y distribución de sus propios contenidos. En este sentido, Castells (2001) reconoce que internet ha impactado la sociedad humana en una medida que es difícil de medir. Almirón y Jarque (2008) señalan que los sucesos más significativos en la comunicación política lo conforman lo acontecido a finales del año 2010 y principios del 2011 en los países árabes de Oriente Medio y el Magreb. Las protestas y manifestaciones de carácter popular y político en Túnez, Egipto, Yemen, Libia o Siria, que provocaron la caída de las dictaduras existentes, fue un acontecimiento al que se denominó La primavera árabe.

Para Klinger y Svensson (2016), así como para Van-Dijck y Poell (2013), las redes sociales permiten que los líderes y partidos políticos se constituyan en comunicadores políticos directos, ser sus propios intérpretes y eludir la intermediación de los medios tradicionales (radio, televisión y prensa escrita). En gran medida, aunque no de manera total, esto se da teniendo en cuenta que el fenómeno de lo político en internet es una cuestión líquida y no sólida. Bauman (1999) señaló este aspecto al decir que es una cuestión que se ha disuelto, debido a que los partidos políticos pierden prevalencia frente al caos de posiciones y posturas polarizadas y el desarraigo de los partidos políticos. 
Se determina como político lo que le interesa al conjunto de opiniones personales que, en el ciberespacio, toma forma de opinión pública tras la contraposición de argumentos y puntos de vista diferentes, al que también podemos llamar espacio público (Habermas, 1981). Así, el espacio público en la actualidad incluye a internet, la gran red que logra interconectar los dispositivos digitales a nivel global (Mazzoleni, 2010). De esta manera, amplía la comunicación humana y da forma a un arma poderosa para la lucha política. En este sentido, Cotarelo (2013) afirma: "cuando ese ámbito digital, universal y sin barreras se presta como contexto de cualquier tipo de acción social, lo llamamos ciberespacio; y cuando esa acción tiene finalidad política, ciberpolítica" (p. 237).

Los cambios que se vienen registrando en la comunicación humana por el surgimiento del ciberespacio constituyen un desafío para una nueva ciudadanía y una nueva democracia con una participación ciudadana más activa en el debate público (Rodríguez Morató, 2007). Y tal como refiere Orihuela (2011), se puede decir que Twitter terminó de completar el círculo que iniciaron los blogs en su momento. Además, agregó interactividad al otorgar la oportunidad de comunicarse entre usuarios o tener como seguidor a alguien sin tener que aceptar una invitación.

Según Lessing (2002), la conducta en el ciberespacio tiene cuatro tipos de restricciones: "1) el código (arquitectura), 2) el mercado, 3) las normas y 4) la ley"; y precisa que "la condición predeterminada en el ciberespacio es la anonimidad" (p. 174). Todo ello viene dando sentido a un nuevo concepto: la ciudadanía digital. Este concepto comprende, entre otros elementos: i) el acceso a la tecnología digital y al ciberespacio; ii) el gobierno electrónico; y iii) una nueva regulación sobre estos como la declaración de derechos humanos en el ciberespacio.

En resumen, Twitter es una red social que opera a nivel del ciberespacio y permite la comunicación entre sus usuarios manera sencilla y breve (con no más de ciento cuarenta caracteres) sin el requisito de ser aceptado previamente, lo que favorece el intercambio de opiniones y puntos de vista.

\section{RESULTADOS}

\subsection{Partidos políticos estudiados}

En la tabla 1 se relacionan los partidos políticos con la indicación de sus siglas y cuentas de Twitter. 
Tabla 1. Partidos políticos estudiados (junio 2019-junio 2020)

\begin{tabular}{lcl}
\hline \multicolumn{1}{c}{ Partidos políticos } & Siglas & \multicolumn{1}{c}{ Cuentas de Twitter } \\
\hline Fuerza Popular & FP & @PFuerzaPopular \\
Frente Ámplio & $F A$ & $@$ BancadaFAperu \\
Partido Aprista Peruano & PAP & @APRA_Oficial \\
Acción Popular & AP & $@$ AccionPopular \\
Alianza para el Progreso & APP & $@$ Peru_APP \\
Contigo & C & $@$ CONTIGO_pe \\
Partido Popular Cristiano & PPC & $@$ ppc_informa \\
Partido Nacionalista & PNP & $@$ pnacionalistap \\
Somos Perú & $S P$ & $@$ SomosP_Oficial \\
Partido Morado & PM & @partidomorado \\
\hline
\end{tabular}

Fuente: elaboración propia.

\subsection{Nivel de adhesión de la población a los partidos políicos}

En la tabla 2 se determina el nivel de adhesión de la población por el número de seguidores que han logrado alcanzar.

Tabla 2. Partidos políticos y adhesión de la población

\begin{tabular}{ccccc}
\hline Partidos políticos & Seguidores (junio 2019) & Seguidores (junio 2020) & Variación & $\%$ \\
\hline FP & 33.700 & 36.500 & 2.800 & 27,26 \\
PNP & 33.900 & 33.800 & $(-) 100$ & 25,24 \\
PAP & 21.700 & 22.700 & 1.000 & 16,95 \\
FA & 9.041 & 10.500 & 1.459 & 7,84 \\
AP & 7.892 & 10.000 & 2.108 & 7,47 \\
PM & 2.836 & 7.919 & 5.083 & 5,91 \\
APP & 6.762 & 7.832 & 1.070 & 5,85 \\
$C$ & 3.623 & 3.797 & 174 & 2,84 \\
SP & 292 & 677 & 385 & 0,51 \\
PPC & 128 & 164 & 36 & 0,12 \\
& & 85.974 & 133.889 & 47.915 \\
\hline
\end{tabular}

Fuente: elaboración propia.

Nota: El PPC en su página @ppc_peru, no registró actividad ni información sobre sus seguidores al 30 de junio de 2020. Por este motivo se optó por estudiar la página alterna @ppc _informa, que sí registra alguna actividad. 


\subsection{Efectividad de los partidos politicos en conseguir nuevos seguidores}

En la tablas 3 (a y b) se determina la efectividad de los partidos según el mayor número de seguidores obtenidos durante el período de estudio (junio 2019-junio 2020).

Tabla 3a. Partidos políticos: incremento adhesión de la población (Twitter)

\begin{tabular}{cc}
\hline Partidos políticos & Incremento de seguidores \\
\hline$P M$ & 5.803 \\
$F P$ & 2.800 \\
$A P$ & 2.108 \\
$F A$ & 1.459 \\
APP & 1.070 \\
$P A P$ & 1.000 \\
SP & 385 \\
C & 174 \\
$P P C$ & 36 \\
$P N P$ & $(-) 100$ \\
\hline
\end{tabular}

Fuente: elaboración propia.

Tabla 3b. Partidos políticos: incremento adhesión de la población (Twitter)

\begin{tabular}{cc}
\hline Partidos políticos & Incremento de seguidores \\
\hline PM & 5.803 \\
$F P$ & 2.800 \\
AP & 2.108 \\
$F A$ & 1.459 \\
APP & 1.070 \\
PAP & 1.000 \\
SP & 385 \\
$C$ & 174 \\
$P P C$ & 36 \\
$P N P$ & $(-) 100$ \\
\hline
\end{tabular}

Fuente: elaboración propia.

\subsection{Nivel de producción informativa de los partidos políticos en Twitter}

En la tablas 4 (a y b) se determina el nivel de producción informativa de los partidos políticos por el número de publicaciones totales registradas al mes de junio de 
2020 (producción acumulada), así como por el número de publicaciones en el mes de estudio (producción relativa).

Tabla 4a. Producción informativa acumulada al 30 junio 2020 (Twitter)

\begin{tabular}{ccc}
\hline Partidos políticos & Tweets producidos & $\%$ \\
\hline FA & 17.300 & 29 \\
PAP & 15.200 & 25 \\
PNP & 11.100 & 19 \\
APP & 7.614 & 13 \\
AP & 3.156 & 5 \\
C & 2.853 & 5 \\
FP & 2.257 & 4 \\
PM & 261 & 0 \\
SP & 127 & 0 \\
PPC & 90 & 0 \\
& 59.958 & 100 \\
\hline
\end{tabular}

Fuente: elaboración propia.

Tabla 4b. Producción informativa relativa del mes de estudio (Twitter)

\begin{tabular}{ccc}
\hline Partidos políticos & Tweets producidos & $\%$ \\
\hline APP & 27 & 30 \\
$C$ & 24 & 26 \\
FA & 15 & 16 \\
PNP & 15 & 16 \\
$F P$ & 5 & 5 \\
$P M$ & 5 & 5 \\
PAP & 0 & 0 \\
AP & 0 & 0 \\
SP & 0 & 0 \\
PPC & 0 & 0 \\
& 91 & 100 \\
\hline
\end{tabular}

Fuente: elaboración propia.

\subsection{Nivel de efectividad de los Tweets emitidos}

En la tabla 5 se determina el nivel de efectividad por los tweets que obtuvieron el mayor número de "me gusta". 
Tabla 5. Partidos políticos: efectividad de los mensajes producidos (Twitter)

\begin{tabular}{|c|c|c|c|c|c|}
\hline Partidos políticos & Me gusta & Comentarios & Compartidos & $\%$ de me gusta & \\
\hline $\mathrm{FP}$ & 3.778 & 2.304 & 1.459 & 33,40 & \\
\hline PM & 2.872 & 392 & 1.072 & 25,39 & \\
\hline PNP & 1.418 & 278 & 544 & 12,54 & \\
\hline FA & 1.764 & 240 & 602 & 15,60 & \\
\hline C & 952 & 513 & 409 & 8,42 & \\
\hline APP & 527 & 88 & 145 & 4,66 & \\
\hline PAP & 0 & 0 & 0 & 0,00 & \\
\hline AP & 0 & 0 & 0 & 0,00 & \\
\hline PPC & 0 & 0 & 0 & 0,00 & \\
\hline SM & 0 & 0 & 0 & 0,00 & \\
\hline Totales & & 11311 & 3,815 & 4231 & 100.00 \\
\hline
\end{tabular}

Fuente: elaboración propia.

\subsection{Tipo de mensajes que obtuvieron mayor aprobación en el ciberespacio}

Se hace un análisis a partir del mensaje que obtuvo mayor cantidad de "me gusta" en cada uno de los partidos políticos estudiados.

\section{Partido Morado}

Fecha: 29 de diciembre de 2019. Tweet: "El congreso disuelto impidió investigación a involucrados en casos Lava Juez y Cuellos Blancos. En enero de 2020, los peruanos tendremos la oportunidad de elegir un nuevo congreso para luchar frontalmente contra la corrupción e impunidad. El voto de cada peruano marcará la diferencia" (Partido Político Partido Morado, 2019). Obtuvo: 2.200 me gusta, 306 comentarios y 911 compartidos.

\section{Fuerza Popular}

Fecha: 11 de octubre de 2019. Tweet del pronunciamiento de Keiko Fujimori: "puedo haber cometido aciertos y desaciertos, pero si lo hice, fue pensando que era lo correcto en su momento y asumo el 100\% de la responsabilidad sobre mis actos y decisiones políticas" (Partido Político Fuerza Popular, 2019). Obtuvo: 1.100 me gusta, 1.300 comentarios y 443 compartidos.

\section{Frente Amplio}

Fecha: 27 de marzo de 2020. Tweet: "Bancada FA reafirma su compromiso de la lucha contra la corrupción y rechaza toda forma de transfuguismo. Seguiremos luchando contra la corrupción, defendiendo la justicia y ecología, pero, sobre todo, combatiendo contra la cultura patriarcal y machista" (Partido Político Frente Amplio, 2020). Obtuvo: 732 me gusta, 97 comentarios y 208 compartidos. 


\section{Partido Nacionalista}

Fecha: 01 de octubre de 2019. Tweet: "Durante la gestión nacionalista se depuró todo vestigio fujimontesinista de las FFAA" (Partido Político Partido Nacionalista, 2019). Obtuvo: 395 me gusta, 36 comentarios y 86 compartidos.

\section{Partido Contigo}

Fecha: 25 de noviembre 2019. Tweet: "Pronunciamiento del Fundador y candidato por Lima Juan Cheput "más allá de discrepancias políticas, celebramos que la jurisprudencia se imponga por encima de las pasiones y emociones [...] contigo es democracia contigo es institucionalidad" (Partido Político Partido Contigo, 2019). Obtuvo: 265 me gusta, 83 comentarios y 70 compartidos.

\section{APP}

Fecha: 19 de abril 2020. Tweet: "Invitación al primer foro político virtual, en el cual se charlará sobre el retiro del 25\% de las AFP" (Partido Político Alianza para el progreso, 2020). Obtuvo: 104 me gusta, 16 comentarios y 31 compartidos.

\subsection{Objetivos aparentes de los mensajes de los partidos políiticos}

Determinada entre las alternativas: obtener poder, mantener poder o ejercer poder. Nuestra evaluación arroja: ejercer el poder (FP); obtener poder (FA, APP, C, PNP y PM); en tanto no registraron actividad oficial (PAP, AP, PPC y SP).

\subsection{Lucha entre los partidos políticos estudiados}

En la tabla 6 se muestra la lucha entre los partidos políticos determinada por las posiciones a favor o en contra, acuerdo o desacuerdo, crítica o apoyo hacia otras organizaciones políticas.

Tabla 6. Cuadro de conflictividad de los partidos políticos

\begin{tabular}{|c|c|c|c|c|c|c|c|c|}
\hline Lucha política & $\mathrm{FP}$ & PAP & $\begin{array}{c}\text { Gobierno de } \\
\text { Vizcarra }\end{array}$ & $\begin{array}{c}\text { Directiva } \\
\text { congreso disuelto }\end{array}$ & $\begin{array}{l}\text { Alan } \\
\text { García }\end{array}$ & $\begin{array}{l}\text { Kaiko } \\
\text { Fujimori }\end{array}$ & $\begin{array}{l}\text { Gino } \\
\text { Costa }\end{array}$ & $\begin{array}{l}\text { Fiscalía } \\
\text { Nación }\end{array}$ \\
\hline$F P$ & & & $X$ & & & & & $X$ \\
\hline$F A$ & & & $X$ & $X$ & & & & \\
\hline \multicolumn{9}{|l|}{ PAP } \\
\hline \multicolumn{9}{|l|}{$A P$} \\
\hline$A P P$ & & & $X$ & & & & & \\
\hline C & & & & & & & $X$ & \\
\hline \multicolumn{9}{|l|}{$P P C$} \\
\hline PNP & $X$ & $X$ & $X$ & & $X$ & $\mathrm{X}$ & & \\
\hline \multicolumn{9}{|l|}{$S P$} \\
\hline$P M$ & & & & & & & & \\
\hline
\end{tabular}

Fuente: elaboración propia. 


\section{DISCUSIÓN DE RESULTADOS}

1) Partidos políticos estudiados

Se estudiaron los diez partidos políticos más importantes a juicio del investigador en el mes de junio de 2019: Fuerza Popular (FP), Frente Amplio (FA), Partido Aprista Peruano (PAP), Acción Popular (AP), Alianza para el Progreso (APP), Contigo (C), Partido Popular Cristiano (PPC), Partido Nacionalista (PNP), Somos Perú (SP), Partido Morado (PM).

2) Nivel de adhesión de la población

Los partidos políticos que gozan de mayor adhesión de la población, según sus cuentas de Twitter al mes de junio de 2020 son: 1) Fuerza Popular con 36.500 seguidores que comprende el 27,26\% del total de la muestra; 2) Partido Nacionalista, con 33.800 seguidores que alcanza el 25.24 \%; 3) Partido Aprista Peruano con 22.700 seguidores y el $16,95 \%$ del total de la muestra de estudio. Destaca como el único partido que ha perdido seguidores a junio de 2020 es el Partido Nacionalista (-100 seguidores comparados con el año 2019).

3) Efectividad de los partidos políticos en conseguir nuevos seguidores

El Partido Morado obtuvo el mayor número con 5.803 nuevos seguidores en el periodo de estudio. En segundo lugar, Fuerza Popular tuvo 2.800. El tercero, Acción Popular, tuvo con 2.108.

4) Nivel de producción informativa

En la producción acumulada (todo el tiempo), el Frente Amplio registra la mayor producción informativa en Twitter, con 17.300 publicaciones y $29 \%$ del total estudiado. Sin embargo, este esfuerzo comunicativo no le ha asegurado el primer lugar en cuanto al número de seguidores donde aparece en el cuarto puesto. En el segundo lugar aparece el Partido Aprista con 15.200 publicaciones que cubre el $25 \%$ del total de publicaciones. En el tercer lugar, el Partido Nacionalista tiene 11.100 tweets que alcanza el $19 \%$ del total de publicaciones estudiadas. El partido con mayor cantidad de seguidores, Fuerza Popular, que tiene 36.500 adeptos, solo produjo 2.257 tweets, es decir, el $4 \%$ de la producción informativa. Este resultado es contrario a la lógica de que a mayor esfuerzo comunicativo debería corresponder mayor número de seguidores.

La producción informativa relativa (solo del mes de estudio) muestra como los partidos políticos más dinámicos a: Alianza para el Progreso cuenta con veintisiete tweets; Contigo, veinticuatro tweets; el Partido Nacionalista y el Frente Amplio empatan en el tercer lugar con quince tweets cada uno. En perspectiva, 
el Frente Amplio, el partido con mayor producción informativa relativa, mantiene un ritmo sostenido en su producción informativa. Mientras tanto, Alianza para el Progreso y Contigo vienen desplegando un mayor esfuerzo por ganar espacio en las preferencias de la población. Por otro lado, el partido con mayor cantidad de seguidores, Fuerza Popular, registra una baja actividad de publicaciones oficiales.

5) Nivel de efectividad de los Tweets emitidos

Fuerza Popular es el que tiene mayor aceptación en el ciberespacio, ya que registra 3.778 me gusta en sus tweets emitidos, 2.304 comentarios y 1.459 compartidos o reenviados. El segundo lugar lo tiene el Partido Morado con 2.872 me gusta, 392 comentarios y 1.072 reenviados. El tercer lugar lo ocupa el Partido Nacionalista con 1.418 me gusta, 278 comentarios y 544 compartidos. No son los partidos políticos más activos, como Alianza por el Progreso con veintisiete publicaciones, Contigo con veinticuatro publicaciones, Frente Amplio y Partido Nacionalista con quince publicaciones, los que han logrado mayor efectividad. Con solo cinco publicaciones Fuerza Popular ha sido el ganador en cuanto a la adhesión de la población, que se puede considerar un logro importante si se considera que es el partido más combatido por otras organizaciones políticas.

6) Tipo de mensajes que obtuvieron mayor aprobación

El Partido Morado viene destacando por su alta actividad en Twitter y ha logrado incrementar el número de seguidores y de aceptación en sus mensajes. Durante el período de estudio tuvo la publicación que logró mayor aceptación (2.200 me gusta). El mensaje era una denuncia al congreso disuelto por haber impedido las investigaciones de corrupción como el caso de lava juez y cuellos blancos². Podemos decir que es un mensaje de carácter político que toma distancia de la corrupción y acusa, al mismo tiempo, a los partidos tradicionales, a los que hace responsables de los problemas del país. Aprovecha su posición de ser un partido nuevo.

El segundo mensaje que gusto más a la población en el Twitter, con 1.100 me gusta, fue el de la lideresa Keiko Fujimori de FP, quien hizo un mea culpa al reconocer que pudo haber cometido aciertos y desaciertos. De esta manera, precisó que lo que hizo fue pensando que era lo correcto en su momento, pero mostró su disposición a asumir el 100 \% de la responsabilidad de sus actos y decisiones políticas. Este es un mensaje de carácter político que busca respaldo emocional a sus decisiones que, para muchos, pudieron ser equivocadas, porque contribuyeron a la crisis política que llevó al cierre del Congreso el 30 de septiembre de 2019.

2 Lava juez y cuellos blancos son las etiquetas con las que se conoce a dos casos de corrupción judicial en el Perú en los que estarían comprometidos magistrados de alta jerarquía, empresarios y algunos políticos. 
El tercer mensaje que gustó más en el ciberespacio fue del Frente Amplio con 732 mensajes. La "Bancada FA reafirma su compromiso de lucha contra la corrupción y rechaza toda forma de transfuguismo. [...] Seguiremos luchando contra la corrupción, defendiendo la justicia y ecología, pero, sobre todo, combatiendo contra la cultura patriarcal y machista". Este es un mensaje de carácter político que busca ser identificado como luchador contra la corrupción, la contaminación ambiental y la cultura patriarcal y machista de la sociedad peruana, que son aspectos sensibles y de gran aceptación por la mayor parte de la población.

En términos generales, podemos señalar que los mensajes políticos que más gustan a la población peruana son los referidos a la lucha contra la corrupción, seguido de asumir las responsabilidades políticas, la defensa ambiental, así como la promoción de una cultura de paz e inclusión social.

7) Objetivos aparentes de los mensajes divulgados

Ejercer poder. Esta es la característica más notoria de Fuerza Popular, pues intentan generar presión sobre el gobierno de turno, lo cual se explica porque, al momento de iniciar el estudio y hasta la disolución del Congreso, era la fuerza política con mayoría en el Congreso de la República.

Obtener poder. Esta es la característica más notoria en los esfuerzos comunicativos de Frente Amplio, Alianza para el progreso, Contigo, Partido Nacionalista y Partido Morado. Se puede notar el esfuerzo que estos partidos vienen realizando por ganarse la adhesión de la población en el contenido de sus mensajes y en su esfuerzo informativo.

No han registrado actividad en sus páginas oficiales el PAP, AP, PPC y SP, lo que puede tener varias interpretaciones: i) están asumiendo un perfil bajo a fin de rehuir el conflicto político directo y para mostrarse neutrales a las acciones del gobierno; ii) hay una crisis organizacional interna por falta de una dirección adecuada. El primer caso se explicaría porque tanto PAP y PPC sufrieron fuertes derrotas en las últimas elecciones, pero no es el caso de AP, que logró ganar las elecciones por el Gobierno metropolitano de Lima (ciudad capital del país). En el caso de SP no se explica esta conducta debido a que mantiene cierta presencia política importante en el Perú con 11 Congresistas en enero de 2020. En el segundo caso, esto devendría en la carencia o deficiencia de un aparato de prensa e imagen (gabinete de comunicación política). En todo caso, faltan datos para poder hacer precisiones más exactas, lo que podría constituir temas para investigaciones futuras.

Podría esperarse que todos los partidos políticos que no están en ejercicio del poder a cargo del Gobierno nacional busquen obtener el respaldo de la población mediante la aprobación de sus propuestas políticas, así como el respaldo a 
la persona de sus candidatos. Además, es posible que los que están en ejercicio del poder político pretendan mantenerlo y ejercerlo a partir de la persuasión la población de lo adecuado de sus decisiones y acciones. Ahora bien, nuestra realidad política demuestra que casi todos los partidos tienen alguna cuota de poder, porque cuentan con representantes en el Congreso de la República, los gobiernos regionales o locales, lo cual determinaría que rehúyen al conflicto evidente.

8) Mapa de conflictividad

Del sentido y la cantidad de los mensajes emitidos en contra de otra organización política, institución o persona, podemos deducir la existencia de lucha política evidente.

1. Fuerza Popular: contra el Gobierno del presidente Martin Alberto Vizcarra Cornejo. De manera accesoria, se descalifica a la Fiscalía de la Nación por una supuesta actuación parcializada.

2. Frente Amplio: contra la Junta directiva del Congreso de la República (disuelta por Decreto Supremo 165-2019-PCM del 30 de septiembre de 2019) y el gobierno de turno.

3. Partido Aprista Peruano: no registra actividad.

4. Acción Popular: no registra actividad

5. Alianza para el Progreso: contra el gobierno de turno, pero solo a través de críticas muy ocasionales.

6. Partido Contigo: contra el Gobierno de Martín Vizcarra, pero de manera discreta; asimismo, contra el congresista Gino Costa Santolalla.

7. Partido Popular Cristiano: no registra actividad

8. Partido Nacionalista: contra el Gobierno de Marín Vizcarra, el expresidente Alan Gabriel Ludwig García Pérez, el Partido Aprista Peruano y Keiko Sofía Fujimori Higuchi (líder de Fuerza Popular).

9. Somos Perú: no registra actividad

10. Partido Morado: no muestra publicaciones que revelen antagonismo o discrepancia directa contra ningún partido ni líder político, ni contra el gobierno de turno. Sus críticas son abiertas y generales y evitan señalar directamente a los adversarios. 


\section{CONCLUSIONES}

Hasta el año 2020, el Perú cuenta con una población estimada en 32.625.948 (INEI, 2020b). Y al igual que sucede en otros países, se viene registrando la predilección del empleo de la red social Twitter para la comunicación política por parte de los partidos y líderes políticos.

Se considera que más del $90 \%$ de los usuarios de internet tienen cuentas de redes sociales en el Perú (Ticona, 2015). Si consideramos que las cifras oficiales del Instituto Nacional de Estadística del año 2019 señalan la existencia de 2.381 .739 de conexiones fijas de Internet y de 26.093.385 de conexiones a internet móvil (INEI, 2020a), podemos concluir que la gran mayoría de peruanos se encuentra interconectados por las redes sociales.

Los resultados del trabajo de investigación nos permiten arribar a las siguientes conclusiones:

La lucha política en el Perú, librada por los partidos políticos en la red social Twitter entre junio 2019 y junio 2020, tuvo un desarrollo asimétrico e irregular, y presentó diferencias significativas entre los diez partidos políticos estudiados. Así, tenemos que en cuanto a la producción relativa de sus mensajes, cuatro partidos no registraron ninguna actividad comunicativa oficial a través de sus páginas de Twitter en el período de estudio: Partido Aprista Peruano (PAP), Acción Popular (AP), Somos Perú (SP) y Partido Popular Cristiano (PPC). Registraron poca actividad con solo cinco mensajes emitidos por Fuerza Popular (FP) y Partido Morado (PM). Tuvieron una actividad mediana con quince mensajes emitidos en el mismo período el Partido Nacionalista (PNP) y el Frente Amplio (FA). Registraron alta productividad informativa Alianza para el Progreso (APP) con veintisiete tweets emitidos y Contigo (C) con veinticuatro mensajes. En resumen: dos partidos muy activos, dos medianamente activos, dos poco activos y cuatro inactivos.

El nivel de adhesión de la población peruana a los partidos políticos, deducida por el número de seguidores en sus cuentas oficiales de Twitter el 30 de junio de 2020, nos muestra bajos niveles de adherencia. Sin embargo, el partido mejor posicionado es Fuerza Popular (FP) con 36.500 seguidores, que cubre el 27,26 \% del total de la muestra de estudio. En segundo lugar, aparece el Partido Nacionalista Peruano (PNP) con 33.800 seguidores y el 25,24 \% de la muestra de estudio. El tercer lugar lo tiene el Partido Aprista Peruano (PAP) con 22.700 seguidores y 16,95\% de la muestra. En el cuarto lugar está el Frente Amplio con 10.500 seguidores, que representa el 7,84 \%. La quinta posición es para Acción Popular con 10.000 seguidores y 7,47 \% del total. El sexto lugar lo tiene el Partido Morado con 7.919 seguidores y el 5,91 \%. El séptimo puesto es de Alianza para el Progreso con 7.832 seguidores y el 5,85 \%. En el octavo puesto figura Contigo, que registra 3.797 seguidores y 2,84 \%. En el noveno lugar está Somos 
Perú, con 677 seguidores y el 0,51 \% del total. En el décimo y último lugar figura el PPC con 164 seguidores y $0,12 \%$. Esto nos permite sugerir a los partidos políticos en general que fortalezcan sus gabinetes de comunicación política para poder lograr un mejor posicionamiento en la sociedad peruana.

Respecto del nivel de efectividad de los partidos políticos para lograr nuevos seguidores, muestra al Partido Morado (PM) como el más efectivo por haber logrado 5.803 nuevos seguidores. El segundo lugar lo ocupa Fuerza Popular (FP), que logró adherir 2.800 nuevos seguidores. En tercer lugar, aparece Acción Popular (AP) con 2.108 nuevos seguidores. Registraron un crecimiento medio el Frente Amplio (FA), con 1.459 nuevos seguidores; Alianza para el Progreso (APP), con 1.070; y el Partido Aprista Peruano (AP), con 1.000 nuevos seguidores. Registraron un bajo incremento Somos Perú (SP), con 385 nuevos simpatizantes; Contigo (C), con 174 nuevos adeptos; y el Partido Popular Cristiano (PPC), con solo 36 nuevos simpatizantes. El Partido Nacionalista (PNP) es el único que decreció en -100 seguidores en el periodo de estudio.

El cuanto al nivel de producción informativa acumulada (todas las publicaciones hasta el 30 de junio de 2020), los partidos políticos estudiados registran en primer lugar al Frente Amplio (FA) con 17.300 publicaciones y el $29 \%$ del total de la muestra; seguido del Partido Aprista Peruano (PAP), con 15.200 publicaciones y el $25 \%$ del total; el tercer lugar es para el Partido Nacionalista (PNP), con 11.100 publicaciones y el $19 \%$ de la muestra; el cuarto lugar lo tiene Acción Popular (AP), con 3.156 y el $5 \%$; en quinto lugar figura Contigo con 2.853 publicaciones y el 5 \% de la muestra; el sexto puesto lo tiene Fuerza Popular con 2.257 publicaciones y el $4 \%$ del total. En términos relativos, la producción informativa del período de estudio coloca en primer lugar a Alianza para el Progreso (APP) con veintisiete tweets emitidos, que cubre el 30 \% de la producción total de la muestra de estudio; seguido de Contigo, con veinticuatro tweets y el $26 \%$ del total; en tercer lugar figuran el Frente Amplio (FA) y el Partido Nacionalista (PNP), que empatan con 15 publicaciones y el $16 \%$ del total de la muestra para cada uno.

El nivel de efectividad de los mensajes publicados por los partidos políticos en el período del estudio ubica en el primer lugar a Fuerza Popular (FP), que obtuvo 3.778 me gusta, lo que constituye el 33,40\% del total de la muestra; en el segundo lugar aparece el Partido Morado (PM) con 2.872 me gusta y el 25,39 \% de la muestra; en tercer lugar aparece el Partido Nacionalista (PNP) con 1.418 me gusta y el $12,54 \%$ del total. Observamos que no son los partidos más activos los que gozan de mayor efectividad. Los datos muestran que son los partidos que han fidelizado mejor a sus seguidores los que obtienen mayor cantidad de me fusta. Esta situación se deduce a partir de los mensajes que obtuvieron mayor cantidad de me gusta. Estos no necesariamente contenían información de propuestas políticas, sino que fueron comunicados 
simples sobre sentimientos personales o familiares de sus líderes, lo que evidencia campañas centradas en el líder y no en programas de gobierno.

Los tipos de mensajes que tuvieron mayor acogida, determinada por los me gusta obtenidos, muestran en primer lugar al Partido Morado (PM). Este mensaje se refiere a la denuncia de corrupción a los partidos tradicionales con sus representantes en el Congreso de la República por haber impedido el combate a la corrupción (investigaciones) y que logró 2.200 me gusta. En segundo lugar, aparece Fuerza Popular (FP) con el mensaje emitido por su líder Keiko Sofía Fujimori Higuchi. En este mensaje reconoce que pudo haber cometido aciertos y desaciertos, pero que lo hizo pensando que era lo correcto en su momento y mostró su disposición a asumir el 100 \% de la responsabilidad de sus actos y decisiones políticas. Obtuvo 1.100 me gusta. El tercer lugar corresponde al Frente Amplio (FA) con un mensaje en el que se compromete a luchar contra la corrupción y que obtuvo 732 me gusta. En el cuarto lugar figura el Partido Nacionalista (PNP) por su anuncio de haber depurado en su gestión todo vestigio de "fujimontesinismo" en las FFAA que obtuvo 395 me gusta. La quinta ubicación corresponde a Contigo (C), por su pronunciamiento por el respeto a la ley y la jurisprudencia con 265 me gusta. La sexta posición corresponde a Alianza para el Progreso (APP) por su invitación al primer foro político virtual con 104 me gusta. Se destacan también los mensajes referidos a la defensa ambiental, la promoción de una cultura de paz e inclusión social.

Respecto a los objetivos aparentes que persiguen los partidos políticos a través de sus mensajes divulgados, hemos deducido que la característica más notoria de Fuerza Popular (FP) es ejercer poder e intentar generar presión sobre el gobierno de turno. Esto se explicaría con que al momento de iniciar el estudio y hasta la disolución del Congreso de la República, este partido era la fuerza política con mayoría en el Congreso de la República. Obtener poder es la característica más notoria en los esfuerzos comunicativos de Frente Amplio (FA), Alianza para el Progreso (APP), Contigo (C), Partido Nacionalista (PNP) y Partido Morado (PM). Se puede notar el esfuerzo de estos partidos por ganar la adhesión de la población y lograr un mayor respaldo ciudadano.

El mapa de conflictividad entre los partidos políticos revela que el partido que más confronta es el Partido Nacionalista (PNP), que evidencia su enfrentamiento contra Fuerza Popular (FP), Partido Aprista Peruano (PAP) y el Gobierno de turno. El segundo partido que más confronta es Fuerza Popular (FP), que evidencia lucha o antagonismo contra el gobierno de turno a cargo del presidente Martín Alberto Vizcarra Cornejo, e incluye al Ministerio Público por su actuación en las investigaciones contra su líder Keiko Sofía Fujimori Higuchi. Lucha moderada muestran Frente Amplio (FA), Alianza para el Progreso (APP) y Contigo (C) contra el gobierno de turno, mediante 
críticas puntuales. El Partido Morado (PM) rehúye la confrontación contra otro partido o líder político, lo que obedecería a una estrategia de crecimiento.

Consideramos que esta investigación debe ser actualizada anualmente, dado que son muchas las características idiosincráticas que inciden en la forma en que se desarrolla la lucha política en Twitter. Resultaría pertinente el estudio de la totalidad de partidos políticos inscritos en el ROP del JNE para tener un panorama más completo de la lucha política a nivel de los partidos políticos peruanos. Proponemos el estudio de la lucha política en el ciberespacio a nivel de los principales líderes políticos del Perú.

\section{REFERENCIAS}

Aguilar, J. (2016). El Partido Predominante en el Municipio de Nezahualcóyotl 1996-2006 [tesis de pregrado]. Repositorio Institucional/RI Universidad Autónoma del Estado de México, Amecameca. http:// ri.uaemex.mx/handle/20.500.11799/64916

Aira, T. (2015). La comunicación política. UOC.

Aparici, R. (2010). Conectados en el ciberespacio. Universidad Nacional de Educación a Distancia, UNED. Almiron, N. y Jarque, J. (2008). El mito digital. Discursos hegemónicos sobre internet y periodismo. Anthropos. Bauman, Z. (1999). Culture as praxis. Sage publications.

Barrio, A. M. (2017). Ciberdelitos: Amenazas criminales del ciberespacio. REUS Editorial.

Bovero, M. (1997). La naturaleza de la política. Poder, fuerza, legitimidad. Universidad de Turín. Artículos y secciones especiales. Revista Internacional de Filosofia Política, (10), 91 102. https://bit.ly/3xsxdaU

Bustelo, E. (1998). Expansión de la ciudadanía y construcción democrática. En E. Bustelo y A. Minujin (eds.), Todos entran. Propuestas para sociedades incluyentes. Unicef /Grupo Santillana.

Canetti, E. (1960). Masa y poder. Ed. Alianza.

Castells, M. (2001). Internet y la Sociedad Red. http://fcaenlinea.unam.mx/anexos/1141/1141_u5_act1.pdf

Chadwick, A. (2017). The hybrid media system. Politics and Power. Second edition. Oxford University Press.

Condori, E. (2017). La práctica oligopólica y el periodismo político en los diarios del Grupo El Comercio en el Perú- 2015. EPG UNMSM.

Cotarelo, R. (2013). Ciberpolítica. Las nuevas formas de acción y comunicación política. Tirant.

Cortés, S. C. E. (2004). Derechos digitales y control del ciberespacio. Revista Latinoamericana de Comunicación Chasqui (86), 66-73. https://www.redalyc.org/pdf/160/16008610.pdf

El Comercio (2019, 21 de octubre). ¿Qué partidos podrían participar si las elecciones se adelantan al 2020? https://bit.ly/2VsOmg7

Habermas, J. (1981). Historia crítica de la opinión pública. La transformación estructural de la vida pública. Gustavo Gili.

INEI (2020a). Conexiones a internet. https://www.inei.gob.pe/buscador/?tbusqueda=internet

INEI (2020b). Población estimada al año 2020. https://bit.ly/31Bb740 
Klinger, U. y Svensson, J. (2016). Network media logic: some conceptual considerations. En A. Bruns, G. Enli y E. Skogerbø, (eds.), The Routledge Companion to Social Media and Politics. Routledge.

Lessig, L. (2002). Las leyes del ciberespacio. Thēmis-Revista De Derecho, (44), 171-179. https://bit.ly/3ywbr7F

López-Rabadán, P., López-Mery, A. y Doménech-Fabregat, H. (2016). La imagen política en Twitter. usos y estrategias de los partidos políticos españoles. Index comunicación. Revista científica de comunicación aplicada, 6(1), 165-195. https://journals.sfu.ca/indexcomunicacion/index.php/indexcomunicacion/ article/view/270

Marcos García, S. (2018). Las redes sociales como herramienta de la comunicación política. Usos políticos y ciudadanos de Twitter e Instagram. [tesis doctoral], Universitat Jaume I. Tesis Doctorals Universidad Jaume I en Xarxa (TDX). https://www.tdx.cat/handle/10803/662817\#page =1

Marreros Núñez, M. A. (2018, 30 de abril). Factores asociados al uso de las redes sociales Facebook y Twitter en las estrategias de marketing político dirigidas a personas votantes entre 18 y 24 años de niveles socioeconómicos A y B en Lima Metropolitana. Itesis de licenciatura, Universidad Peruana de Ciencias Aplicadas (UPC)]. Repositorio Académico UPC. https://doi.org/10.19083/tesis/624296

Mazzoleni, G. (2010). Modelos y efectos de la interacción política-medios. En La Comunicación política. Alianza Editorial.

Mejía, J. (2015). Estrategias de marketing político en Facebook utilizados por el equipo de campaña de no a la revocatoria de la alcaldesa de Lima, Susana Villarán Itesis de licenciatura, Universidad Nacional Mayor de San Marcos]. Cybertesis, Repositorio de Tesis Digitales, https://cybertesis.unmsm.edu.pe/ handle/20.500.12672/4514

Meléndez, C. (2012, 4 de septiembre). Caviares. [mensaje en un blog]. http://jorobadonotredame. blogspot.com/2012/09/caviares.html

Mouffe, Ch. (1999). El retorno de lo político. Comunidad, ciudadanía, pluralismo, democracia radical. Paidós. https://repositorio.unal.edu.co/handle/unal/57004

Orihuela, J. L. (2011). Mundo Twitter. Una guía para comprender y dominar la plataforma que cambió la red. Alienta.

Partido Politico Partido Morado [@partidomorado]. (2019, 29 de diciembre). [tuit]. https://twitter.com/ partidomorado/status/1211468810257096704?lang=es

Partido Político Fuerza Popular [@PffuerzaPopular]. (2019, 11 de octubre). Keiko Fujimori: "Puedo haber cometido aciertos y desaciertos..." [tuit]. https://bit.ly/36HZd08

Partido Politico Frente Amplio [@BancadaFAperu]. (2019, 27 de marzo). [tuit]. https://twitter.com/ bancadafaperu/status/124361 1531327070208

Partido Politico Partido Nacionalista [@BancadaFAperu]. (2019, 01 de octubre). [tuit]. https://twitter. com/pnacionalistap/status/1179144299008741376

Partido Politico Partido Contigo [@CONTIGO_pe]. (2019, 25 de noviembre). Juan Sheputi: "Más allá de discrepancias políticas, celebramos que la jurisprudencia se imponga por encima de las pasiones y emociones" (...) "contigo es democracia contigo es institucionalidad"." [tuit]. https:// twitter.com/contigo_pe/status/1199116741504319488?lang=de

Presidencia del Consejo de Ministros. (2019, 30 de septiembre). Decreto Supremo que disuelve el Congreso de la República y convoca a elecciones para un nuevo Congreso. Diario Oficial. Decreto Supremo n. ${ }^{\circ}$ 165-2019-PCM. https://www.gob.pe/institucion/pcm/normas-legales/306910 165-2019-pcm 
Presidencia del Consejo de Ministros. (2020, 15 de marzo). Decreto Supremo que declara Estado de Emergencia Nacional por las graves circunstancias que afectan la vida de la Nación a consecuencia del brote del COVID 19. Diario Oficial. Decreto Supremo n. ${ }^{0}$ 044-2020-PCM. https://www.gob.pe/ institucion/pcm/normas-legales/460472-044-2020-pcm

Plejánov, G. V. (2017). El socialismo y la lucha política. Alejandría Proletaria. https://www.marxists.org/ espanol/plejanov/1883/1883-socialismoyluchapolitica.pdf

Rodríguez Morató, A (2007). La sociedad de la cultura. Universidad de Barcelona. http://www.educacionyfp. gob.es/dam/jcr:46ee9bf3-2a5a-46df-ac6a-e58db7a8506e/sociedad-cultura.pdf

Ticona, F. y Ramos, M. (2015). Uso de las redes sociales en el Perú. Revista Científica Investigación Andina, 15(2), 7-14. https://revistas.uancv.edu.pe/index.php/RCIA/article/view/13

Van Dijck, J. y Poell T. (2013). Understantinding Social Media Logic. Media and Communication, 1(1), 2-14. https://www.cogitatiopress.com/mediaandcommunication/article/view/70

Velásquez Ramírez, A. (2013). Espacio de lucha política. Teoría política y el giro espacial. Argumentos. Estudios críticos de la sociedad, (73), 175-197. https://argumentos.xoc.uam.mx/index.php/argumentos/ article/view/196

Wellman, B. (2001). Physical Place and CyberPlace: The Rise of Personalized Networking. International Journal of Urban and Regional Research, 25(2), 227-252. 